\title{
A review of brachytherapy physical phantoms developed over the last 20 years: clinical purpose and future requirements
}

\author{
Sarah Wilby, MSc, CSci, MIPEM!', Antony Palmer, PhD',2, Wojciech Polak, PhD',2, Andrea Bucchi, PhD² \\ 'Department of Radiotherapy Physics, Clinical Hematology, and Oncology Centre, Portsmouth Hospitals NHS Trust, Cosham, Portsmouth. \\ United Kingdom, ${ }^{2}$ Department of Mechanical Engineering, Faculty of Technology University of Portsmouth, Portsmouth, United Kingdom
}

\begin{abstract}
Within the brachytherapy community, many phantoms are constructed in-house, and less commercial development is observed as compared to the field of external beam. Computational or virtual phantom design has seen considerable growth; however, physical phantoms are beneficial for brachytherapy, in which quality is dependent on physical processes, such as accuracy of source placement. Focusing on the design of physical phantoms, this review paper presents a summary of brachytherapy specific phantoms in published journal articles over the last twenty years (January 1, 2000 - December 31, 2019). The papers were analyzed and tabulated by their primary clinical purpose, which was deduced from their associated publications.

A substantial body of work has been published on phantom designs from the brachytherapy community, but a standardized method of reporting technical aspects of the phantoms is lacking. In-house phantom development demonstrates an increasing interest in magnetic resonance (MR) tissue mimicking materials, which is not yet reflected in commercial phantoms available for brachytherapy. The evaluation of phantom design provides insight into the way, in which brachytherapy practice has changed over time, and demonstrates the customised and broad nature of treatments offered.
\end{abstract} DOI: https://doi.org/10.5114/jcb.2021.103593

Key words: brachytherapy, phantoms, test object.

\section{Purpose}

This aim of this review paper was to present information on brachytherapy phantoms developed over the last 20 years. It offers a starting point for designing new phantoms, and a source of information on existing phantoms.

A phantom, sometimes called as "test-object", can be defined according to $\mathrm{MeSH}$ (medical subject headings thesaurus, produced by the National Library of Medicine) as a device or object used to enhance imaging techniques or for measuring radiation to evaluate performance, often with properties similar to human tissue [1]. A phantom may be designed to test image quality, check geometric accuracy of the radiation source positions, measure radiation dose, or mimic tissue mechanics.

While considerable growth in the range and quantity of commercial phantoms available for the verification of complex external beam radiotherapy techniques has been seen in the last two decades, the same cannot be said for brachytherapy applications [2]. This could be due to a variety of techniques used within the brachytherapy field worldwide, making it difficult to design generic phantoms, or to a smaller size of brachytherapy commercial market, as compared to external beam. However, there is a vast amount of in-house designed phantoms to achieve pre-defined specific endpoints, created by clinical and research groups.

Lack of choice in commercially available phantoms leads clinical physicists and researchers to either obtain and replicate already existing non-commercial designs, or to design and manufacture new phantoms. The first necessary step before starting designing is to perform a literature search, and to the best of our knowledge, such a review has not been published. There is a sub-section on ultrasound phantoms within a review of recommendations on quality assurance of ultrasound systems used for guidance in prostate brachytherapy [3]. Also, there are brachytherapy evaluations and external beam audits, containing sub-sections on phantoms by Palmer [4] and Pasler [5] for brachytherapy and advanced radiotherapy, respectively. In 2014, Xu et al. reviewed a rapidly growing field of computational phantom development [6]. 
However, virtual phantoms could only be complementary to physical phantoms for brachytherapy, in which quality was dependent on physical processes, such as accuracy of source placement.

This literature review focuses solely on physical phantoms for brachytherapy. The remaining sections of this paper include methodology, results (tabulated overview of phantoms, followed by sections on phantom size/material, phantoms with integrated radiation dosimeters, and commercially available phantoms), discussion with consideration of the future direction of brachytherapy phantoms, conclusions, and references.

\section{Methodology}

Phantoms included in this evaluation were ascertained from a systematic literature review. The electronic databases PubMed and ScienceDirect were searched for: 1. Brachytherapy [Title] AND Phantom [Title]; 2. Brachytherapy [Title] AND Test Object [All Fields]; 3. Brachytherapy [Title] AND (electromagnetic OR EM) [Title] AND Phantom [Title/Abstract]; 4. Brachytherapy [Title] AND Anthropomorphic [Title]. Both searches were time-limited including papers published between January 1, 2000 and December 31, 2019, full journal paper only, and those published in English. The aim of this literature review focused on the design of physical phantoms and sealed brachytherapy sources. Therefore, unsophisticated phantoms, requiring no manufacturing (e.g., water bath or simple stack of solid materials), phantoms not described in detail, virtual/computational phantoms, electronic brachytherapy, thermal brachytherapy, patient-specific phantoms, unsealed sources, and phantoms for imaging quality tests only, with no specific brachytherapy purpose, were excluded from the study. Duplications were removed.

\section{Literature review results}

Key properties of the phantoms found in this literature review are presented in Tables 1-7, along with the reference to the paper, from which the information was extracted [7-129]. Within these tables, the phantoms are grouped by their primary clinical purpose, deduced from their associated publications.

The following results sections cover phantom size/ materials, phantoms with integrated dosimeters, and commercially available phantoms.

\section{Phantom size/material}

Suitable choices for phantom size/material and dosimetry were demonstrated for ${ }^{192} \operatorname{Ir}[130,131]$ and additional brachytherapy sources, such as ${ }^{103} \mathrm{Pd},{ }^{131} \mathrm{Cs},{ }^{125} \mathrm{I},{ }^{169} \mathrm{Yb}$, ${ }^{192} \mathrm{Ir},{ }^{137} \mathrm{Cs}$, and ${ }^{60} \mathrm{Co}[132]$. According to the latter study, for these sources, only plastic water LR (CIRS, Norfolk, USA) has a deviation of less than 3\% when comparing absorbed dose in the phantom material versus absorbed dose in water. While considering only high-dose-rate (HDR) sources, most commercially available phantom materials meet the evaluation criteria that absorbed dose to phantom versus absorbed dose to water must agree within $3 \%$. It is important to note that these conclusions consider only fixed phantom sizes and measurement distances from the source. For example, Sina et al. concluded that for ${ }^{192} \mathrm{Ir}$, a PMMA phantom of radius larger than $10 \mathrm{~cm}$ should be used with water equivalent to $1 \%$ [133]. Table 1 shows phantoms that are used for source measurements or dosimeter evaluation, in which the materials applied are largely restricted to solid water, plastic water, and PMMA. This is also true for the phantoms used in the dosimetric audits listed in Table $2[34,35,36,37]$. These solid materials can be machined to ensure precise geometric placement of applicators and detectors, which are particularly important in brachytherapy because of high-dose gradients. PMMA was used in one third of the phantoms identified in this review. In addition to favorable dosimetric characteristics, PMMA phantoms proved to be inexpensive, relatively easy to shape, and robust enough for its purpose. A much wider variety of materials were used in phantoms imitating the aspects of human anatomy or tissues (Table 3) as well as in phantoms designed for dose verification measurements, in vivo dosimetry, and secondary cancer incidence risks.

Paraffin wax can be easily molded to the required anthropomorphic shape and it has close to water properties, with an atomic number of 6.82 and a density of $0.9 \mathrm{~g} / \mathrm{cm}^{3}$. It is used in breast, esophageal, gynecological, and endoluminal brachytherapy phantoms, primarily for a dose verification $[43,44,45,46]$. These phantoms are not designed for imaging purposes. Polystyrene is a low-cost option, with light weight, which can be cut into simple shapes, and it has been used in breast phantoms [32,112] for quality control of interstitial implants and treatment planning optimization. Polystyrene was suitable for these interstitial techniques because the needles could be easily pushed through the material without the need for machining. Modelling clay and PVC have also been used as moldable materials.

Gelatin is a cheap and easy way of making a soft tissue substitute, where Young's modulus can be adjusted simply by increasing or decreasing the concentration of gelatin in water. A common alternative to gelatin is agar (also known as "agar-agar") or agarose, a purified form of agar. This may be preferable to gelatin, where the phantom is required for magnetic resonance (MR) imaging, in which varying concentration of agar changes in the T2 relaxation properties, making it possible to match different tissue types. De Brabandere et al. described good agreement of MR and computed tomography (CT) imaging characteristics between prostate and their agar prostate phantom [99]. Additionally, agar has been used in combination with glycerol and cellulose particles, where the aim was to mimic the characteristics of prostate tissue on ultrasound (US) [101,102]. Soliman et al. described a solution of manganese chloride II $\left(\mathrm{MnCl}_{2}\right)$ and copper sulfate $\left(\mathrm{CuSO}_{4}\right)$ to simulate the T1/T2 relaxation times of female pelvis [75].

In publications reviewed in this paper, a 3D printing (rapid prototyping) was first mentioned in 2012 when Ryu et al. utilized rapid prototyping to manufacture 


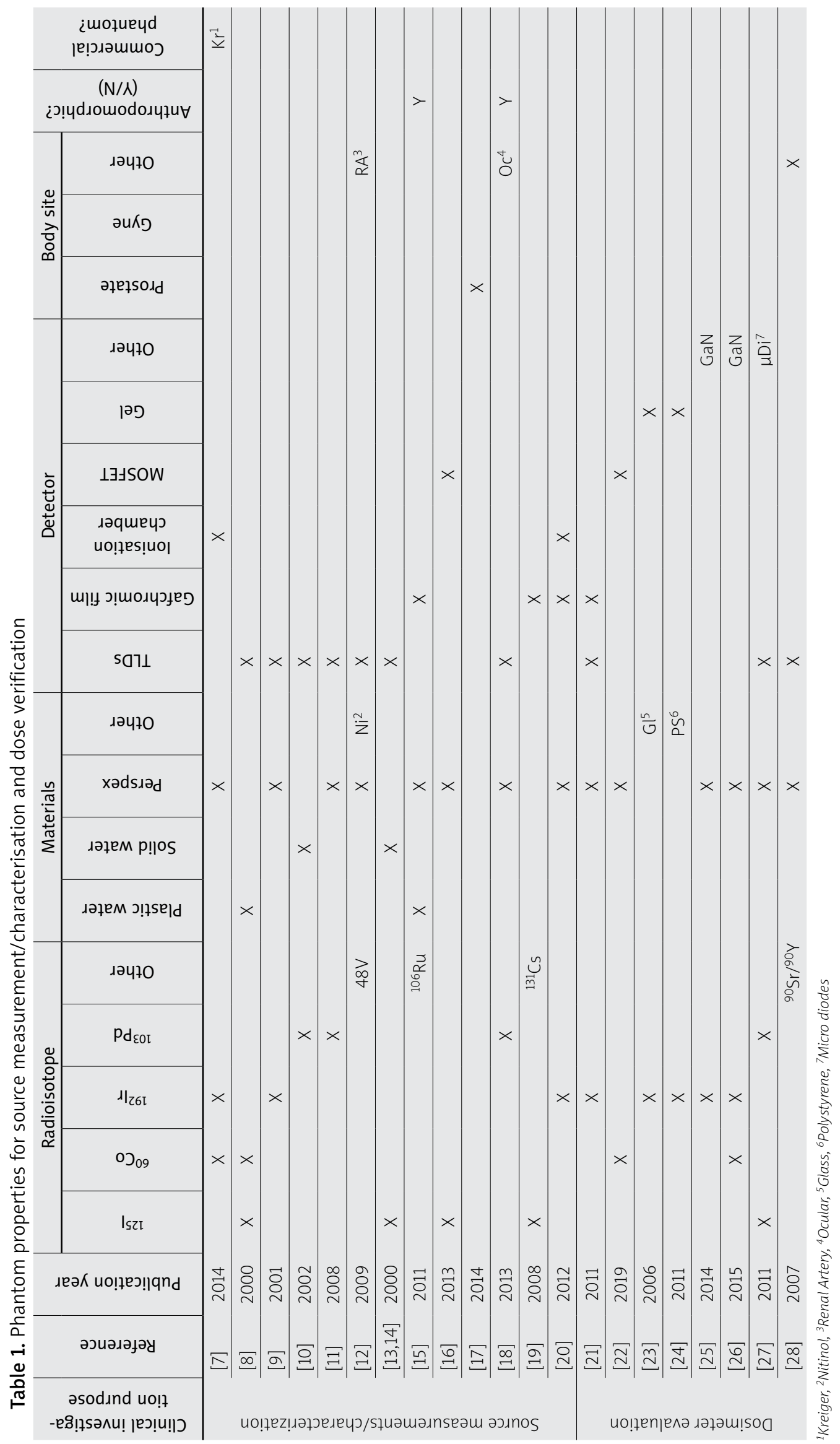




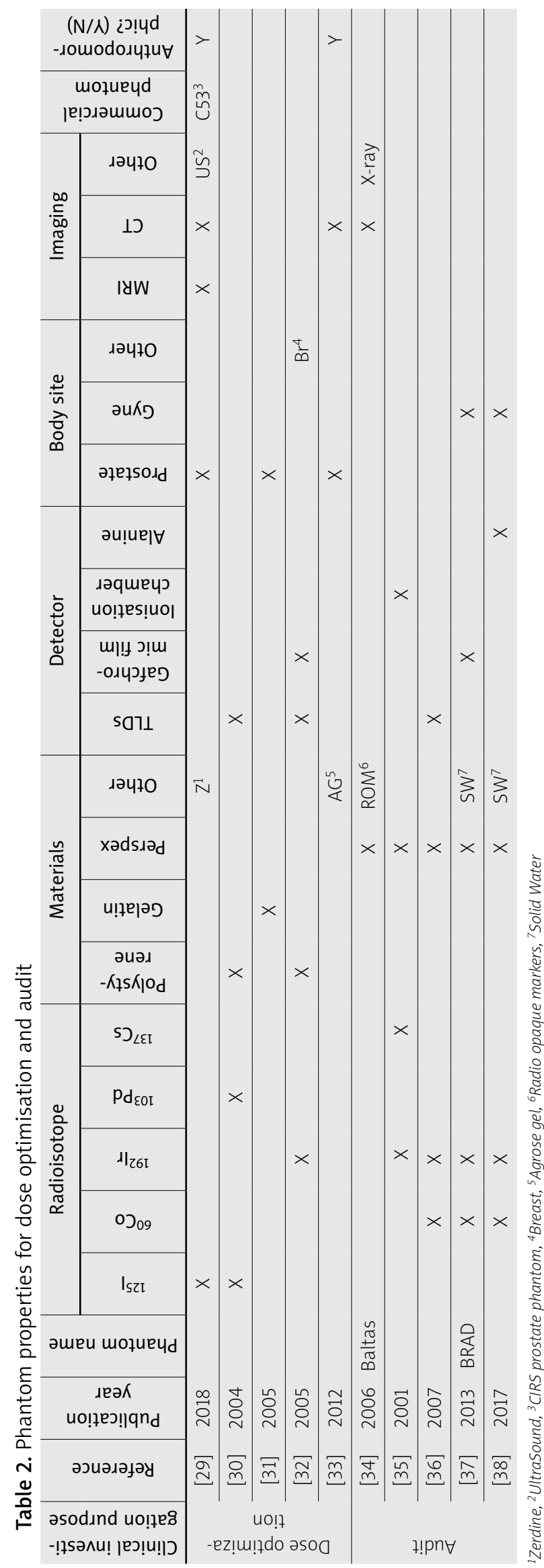

a negative mould for their agar prostate phantom [33]. The concept of 3D printing moulds was also utilized by Nattagh et al. [126] to manufacture a uterus and vagina for their insertion and suturing phantom intended for gynecological brachytherapy training. In 2015, an artificial bladder was directly printed with polylactic acid (PAA) to investigate bladder dose during gynecological brachytherapy [54]. The authors investigated the mass attenuation coefficient of ${ }^{192} \mathrm{Ir}$ for PAA and soft tissue, concluding equivalent behavior in the energy region of interest. Lugez et al. [86] employed rapid prototyping with a resolution of $0.25 \mathrm{~mm}$ to manufacture a phantom consisting of seven HDR prostate brachytherapy grids linked together, with a slot for an EM sensor. This allowed to evaluate the accuracy of tracking catheter paths. In 2019, PAA was used again as a 3D printing material by Leong et al. [110] in their liquid-based single phantom solution for TG128 brachytherapy ultrasound QA [134].

\section{Phantoms with integrated radiation detectors}

Less than half of the articles reviewed referred to phantoms designed specifically for the measurement of radiation. Thermoluminescent dosimeters (TLDs) were the only dosimeters that have been used throughout the period of this review. It was not until 2008, that the use of Gafchromic film in brachytherapy phantoms became more consistent, and dosimetric techniques were established (Tables 1-3 and 6). It is common to see phantoms designed for both TLD's and Gafchromic film, where one acts as verification or gold-standard for the other (Tables 1-3), particularly in the initial evaluation and characterization stage of a new dosimeter.

Seven publications of phantoms designed for source characterization with TLDs had referred to an earlier design of Meigooni et al. [135]. The arrangement of TLDs was chosen to minimize interchip effects, and solid water was selected for water equivalence. Full scatter conditions were met by ensuring at least $10 \mathrm{~cm}$ of solid water between any TLD and the exterior of a phantom. Measurements were performed and recorded following the AAPM TG43 recommendations [136,137,138].

First indication of brachytherapy phantoms using MOSFET's (metal-oxide-semiconductor field-effect transistor) for radiation measurements were found in a 2009 reference [58]. MOSFETs are advantageous for in vivo measurements because their small size and cable assembly allows for needles and catheters insertion, and hence their use in an assessment of prostate, gynecological, and head and neck techniques (Table 3). One of the characteristics of MOSFET showed in the measurements of lowdose-rate (LDR) prostate brachytherapy by Bloemen van Gurp et al. was the angular dependence in a PMMA phantom, which they found to be up to $3.1 \%( \pm 0.51 \%)$ [58]. To overcome this phenomenon, Gambarini et al. proposed the technique of coupling two MOSFET face-to-face detectors in their phantom [59].

In 2006, Hurley et al. described a phantom design for dosimetry of HDR brachytherapy source using a normoxic high-resolution polymer gel dosimeter called "MAGIC" (methacrylic and ascorbic acid in gelatin initiated by cop- 


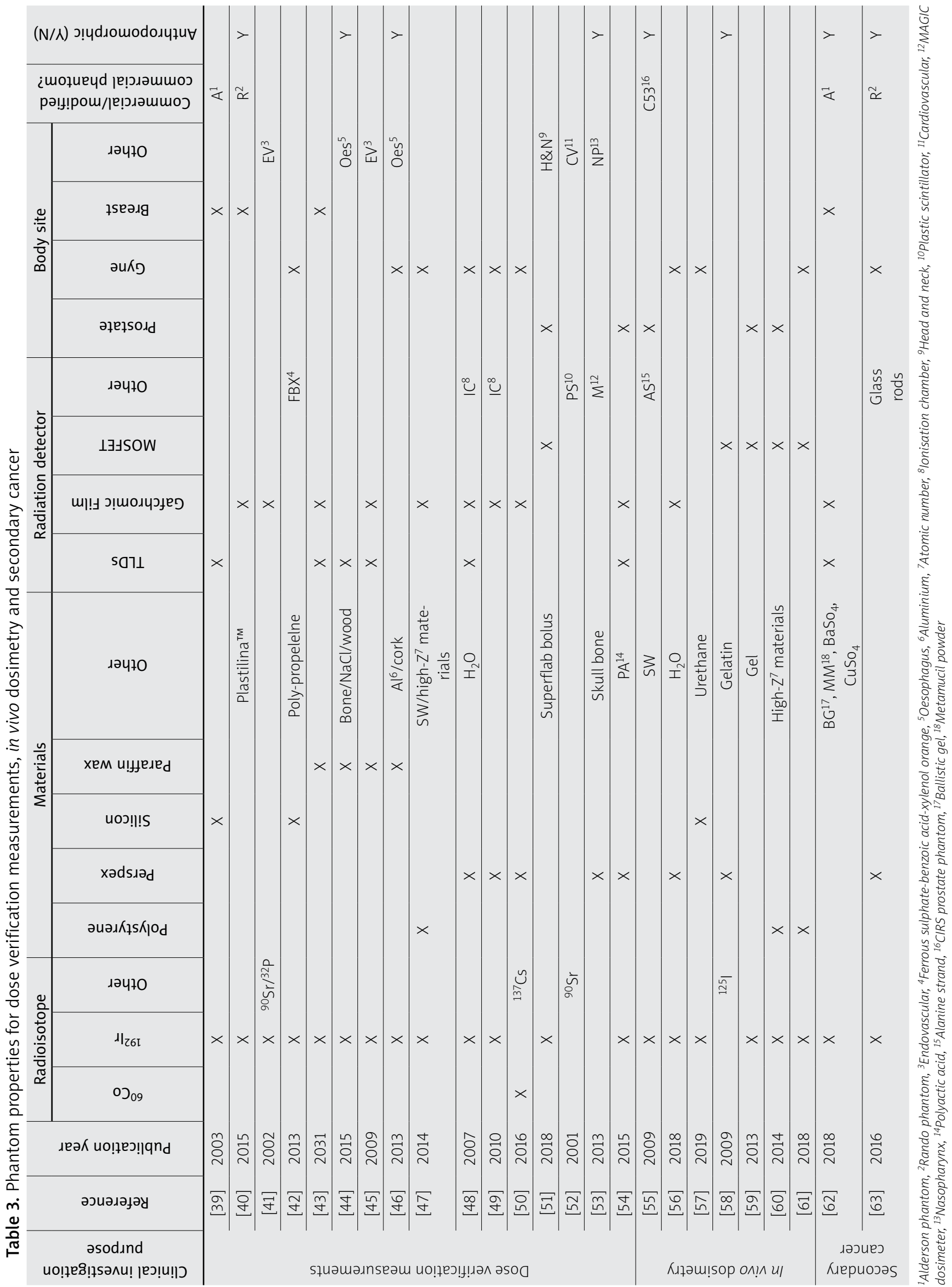




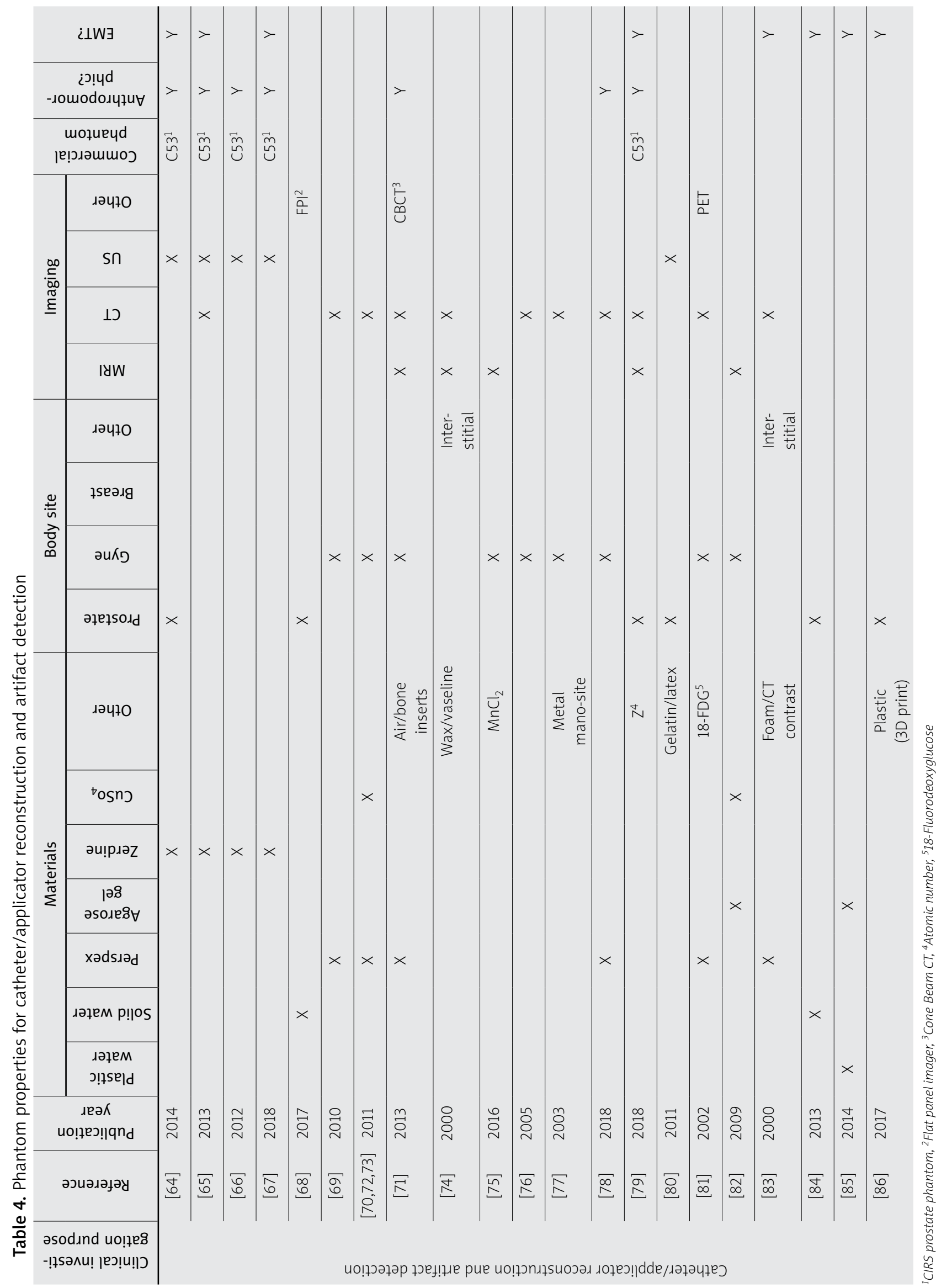




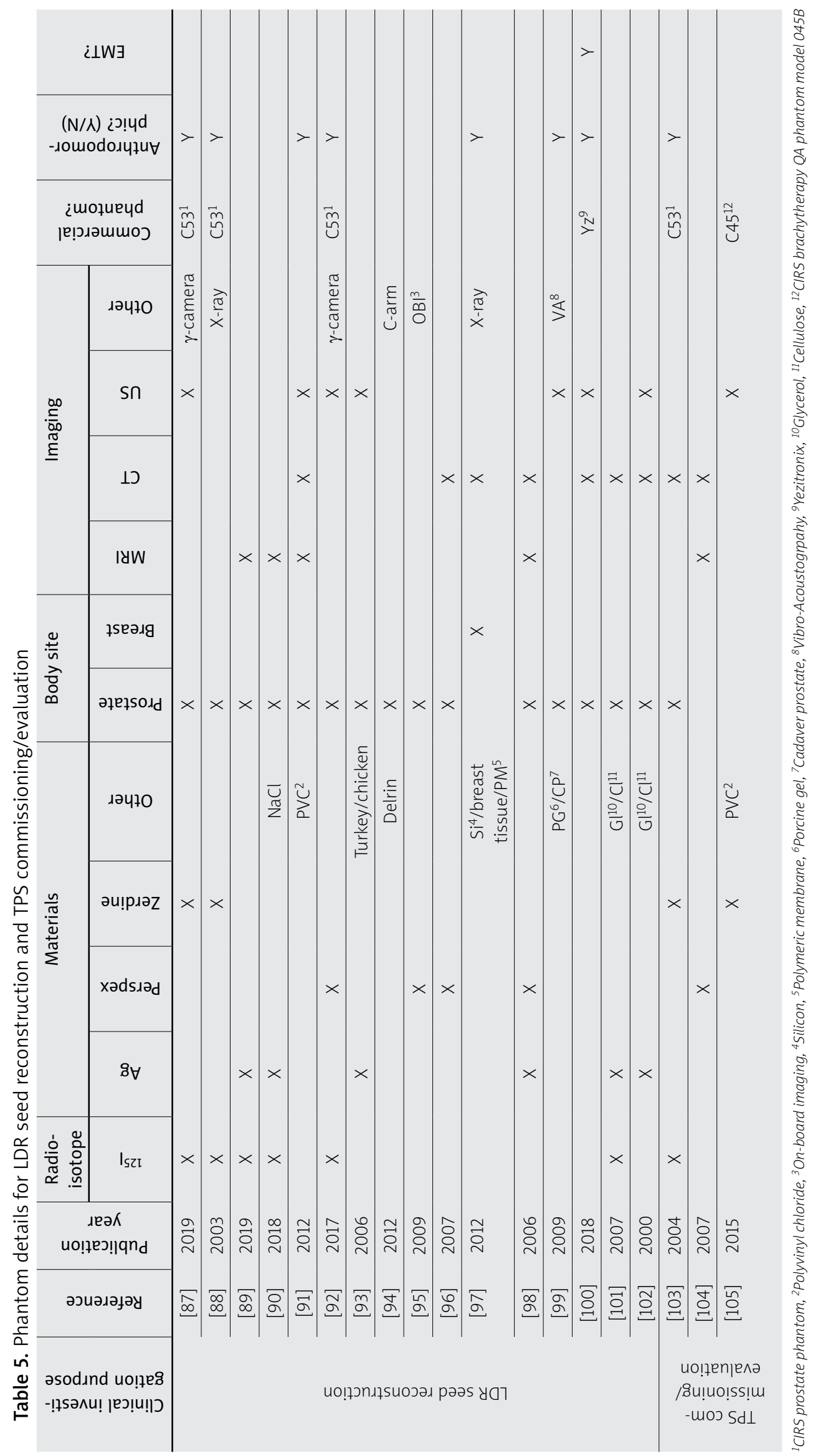




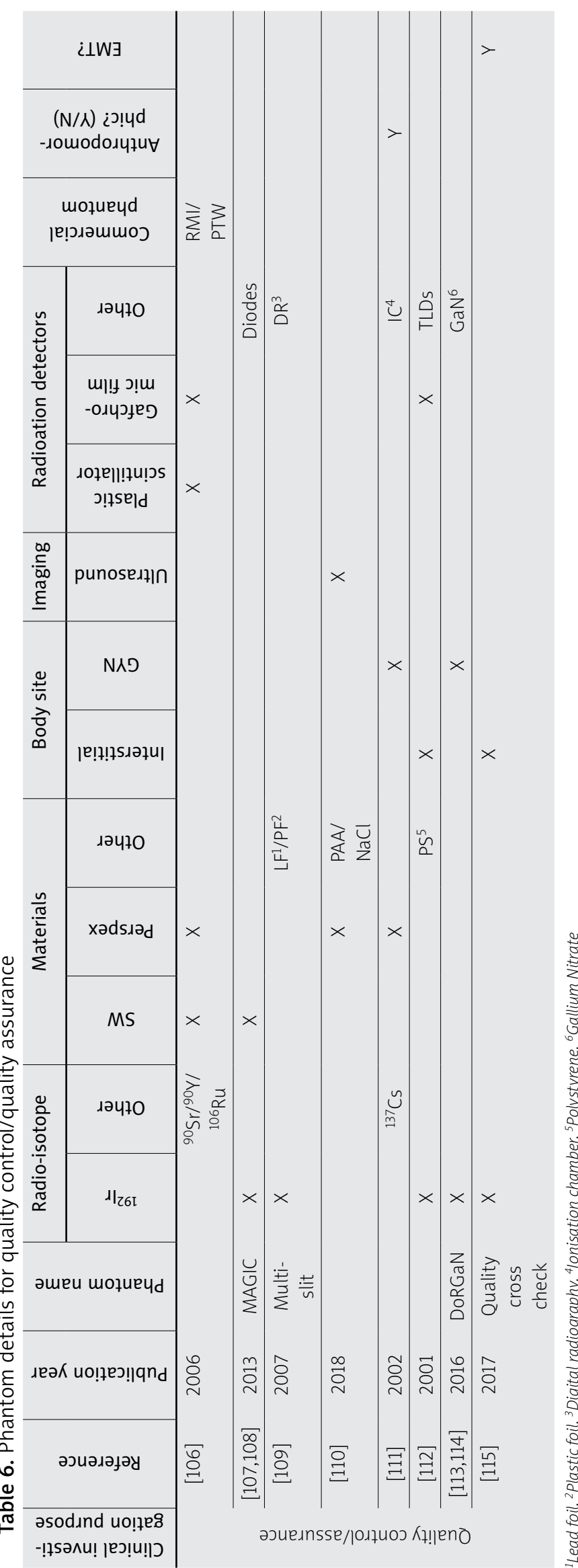

per) combined with high-resolution MRI for dose readout [23]. A variation of MAGIC, named "MAGICA" was used in a nasopharynx phantom design. It varies subtly from the MAGIC recipe by including $0.5 \%$ agarose in the mixture [53]. Fricke gel was used in a phantom design by Carrara et al. In their design, two polystyrene templates with a regular grid pattern were used to hold fifteen catheters in place. Five of these were source catheters and the other ten surrounding them catheters contained radiochromic tissue-equivalent Fricke gel. The structure was placed in water for backscatter [24].

In 2009, a research group from France published [139] the first of a series of papers on implantable real-time dosimetric probes, which used a semiconductor material GaN (gallium nitrate) as a radiation detector, coupled with an optical fiber for radioluminescence collection and transmission. From this work and from a part of the DoRGan research project, an instrumented phantom for QA in HDR brachytherapy, along with an instrumented gynecological applicator was developed. The bulk of the phantom was made from PMMA (120 mm diameter) and incorporated $4 \mathrm{GaN}$-based dosimeters. An insert to the PMMA cylinder allowed for a connection of up to six treatment catheters. The phantom has a great potential as a routine QA system for brachytherapy, with testing showing deviations between the planned and measured dwell positions of $0.11 \pm 0.70 \mathrm{~mm}(1 \sigma)$. The detected ratio for dwell position errors was $96 \%$ at $1 \mathrm{~mm}$, and reached $100 \%$ at $1.5 \mathrm{~mm}$ and beyond. The detected ratio for dwell time errors was $90 \%$ at $0.2 \mathrm{~s}$, and $100 \%$ at $0.3 \mathrm{~s}$ and beyond $[25,26,113,114]$.

Of the publications included in this review, diodes in brachytherapy phantoms were not described until 2011. This was when Broisman and Shani [27] considered the application of spherical micro diodes for brachytherapy dosimetry of LDR ${ }^{125} \mathrm{I}$ and ${ }^{103} \mathrm{Pd}$ sources. Advantages of this solution came from small size diodes $(1.8 \mathrm{~mm}$ diameter), causing little perturbation of the dose and from a $4 \Pi$ symmetry, with a potential for isotropic dosimetry. To characterize the spherical diodes, a range of PMMA phantoms were designed to hold a seed and diodes at fixed positions. Importantly, this paper demonstrates a $4 \Pi$ spherical symmetry in both the axial and azimuthal directions. Espinoza et al. focused on diode utilization for pre-treatment QA in HDR brachytherapy, with the design of a two-dimensional diode array phantom, socalled "magic phantom" [107]. It consisted of an $11 \times 11$ array of silicon p-type diodes with solid water above and below, enabling twenty catheters to be connected. The intended use was the reconstruction of a real-time source position within the phantom, according to the prescribed treatment plan. A further publication on the magic phantom was published two years later in 2015 [108], where additional software was created to compare dwell positions and dwell times measured with the planned treatment from the TPS. This paper also introduces the concept of a new metric called "position-time gamma index" to quantify the quality of delivered plan from the original treatment plan. 


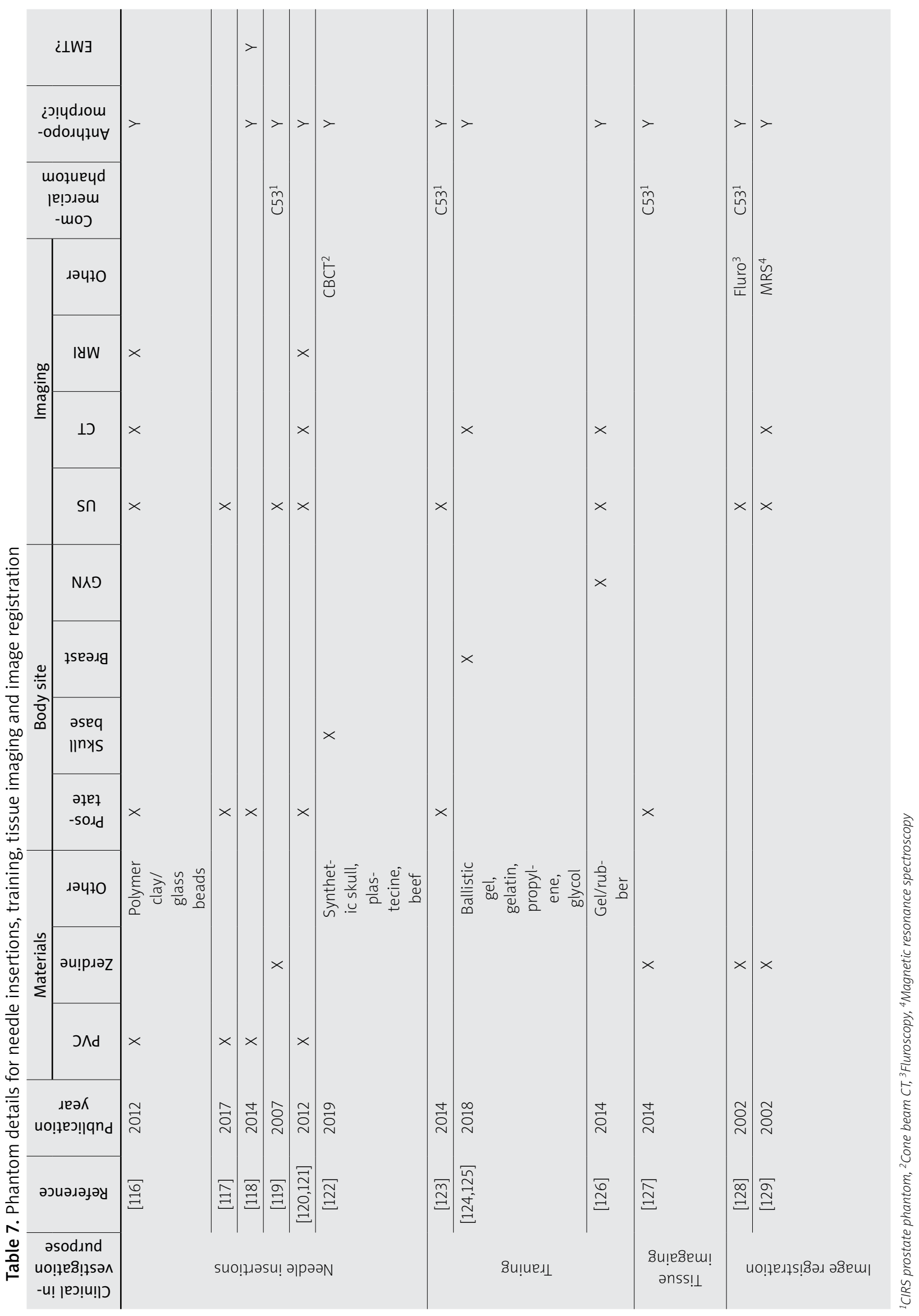




\section{Commercially phantoms}

Of the papers reviewed, only $15 \%$ utilized a commercial phantom. The most common was CIRS (Computerized Imaging Reference Systems, Inc., Norfolk, VA) prostate phantom, model 053 (Tables 2-5 and 7) [140]. From the literature, it appears that it could have started as an inhouse phantom built with a CIRS specification for a study on combining MR spectroscopy with US/CT for prostate brachytherapy [129]. The Yezitronix prostate phantom is a direct competitor for the CIRS model; however, it was described in only one publication identified in this review [100], which may be due to its relatively recent release on the market. Other commercial phantoms mentioned in the reviewed articles included Kreiger phantom [7] for source measurements, Baltas phantom [34] for quality assurance in reconstruction techniques, Rando and Alderson phantoms modified for brachytherapy purposes $[39,40,62,63]$, and CIRS 045 brachytherapy QA phantom for quality assurance in prostate US imaging $[105,110]$.

\section{Discussion}

Iridium-192 is clinically the most used HDR brachytherapy source, and prostate and gynecological brachytherapy are two of the most common techniques (Tables 1-7). It was therefore not surprising that the results of this literature review showed most phantoms developed for these purposes. More surprising was the continued use of TLDs throughout the twenty-year period (Tables 1-3), despite a variety of dosimeters available on the market. TLDs are considered a reliable and validated method for dosimetry in brachytherapy due to their flat energy response and high sensitivity. However, they are labor-intensive in preparation and are disturbed by artifacts, such as volume averaging, self-attenuation, and positioning errors [18]. The uptake of Gafchromic film was unexpectedly slow, considering its less-labor intensive property than TLDs. It had been shown to be ideal for the measurement of dose distributions in regions of changing energy spectra and high-dose gradients as early as in 1991 [141]. Here, the dosimeter chosen for each phantom has a direct effect on the measurement result and therefore must be chosen with full consideration of its purpose. An example is a glass dosimeter being used because it was shown to be more reproducible than TLD's; however, there was no discussion of its high atomic number $(Z=12)$, density $\left(p=2.61 \mathrm{~g} / \mathrm{cm}^{3}\right)$, and angular dependence of $8 \%$ compared to $3 \%$ for TLDs, which could have affected the brachytherapy results [63].

Deformable 3D dosimeters are of interest in brachytherapy phantoms. However, further research is needed to develop gel dosimeters, which have suitable mechanical properties and can measure accurate dose when interstitial techniques are used, particularly considering the evidence that infiltration of oxygen may inhibit the polymerization process [23]. If these issues could be resolved and a practical workflow established for reading out the dose in a clinical department, then is a great potential for 3D gel dosimeters used in brachytherapy phantoms. At present, publications on gel dosimetry focused on characterizing the dosimeters and establishing read-out techniques rather than phantom designs. This was summarized in a study by Farhood et al. [142], a systematic review paper on clinical applications for polymer gel dosimeters in radiotherapy.

Almost half of the US phantom studies used the CIRS prostate phantom. This was probably due to easy commercial availability of this phantom, which increased the number of studies performed with US, since this was the primary imaging modality, for which the phantom was designed. Excluding these studies, the next highest frequency of phantoms designed for imaging were those for MRI. This may reflect the change in imaging practice for gynecological brachytherapy from orthogonal imaging to 3D imaging with CT, CT and MRI-fused, and ultimately, MRI only. A similar transition is true for LDR prostate brachytherapy, from single US imaging, to MRI and USfused, and potentially, MRI only.

Currently, ultrasound QC for prostate brachytherapy tends to be performed with the CIRS brachytherapy phantom; however, this is not suitable for inserting needles and therefore cannot be used for the complete QC as recommended by the AAPM Task Group 128 or the later GEC-ESTRO/ACROP recommendations published in 2020 [143]. The phantom design of Leong et al. has a potential to simplify this QC process, and is relatively simple for clinical users to manufacture. Progress in designing phantoms to digitize QA and QC can be seen in the magic and multi-slit phantoms. Although there is a clear benefit in terms of reduced use of consumables and potential time saving, the equipment must also be practical. There is a concern regarding the multi-slit phantom design and lack of availability of digital radiography within the afterloader room. Phantoms designed for use with electromagnetic tracking should also be included as potential QA tools as demonstrated by Kellermeier et al. [115] and Damato [85]. The potential benefit for this technology in brachytherapy is considerable, as discussed by Tanderup et al. in their paper outlining prospects of technology innovation [2].

A detailed explanation behind the reason for the phantom's design can be overlooked in the publications, and it seems that this is particularly the case when it comes to the choice of materials. For example, the use of MAGIC-A, where $0.5 \%$ agarose has been added to the mixture without any explanation of its purpose. Most commonly, agarose is used to thicken a liquid or for modifying relaxation properties for MRI [53]. An important discrepancy was identified by Zhu et al. in the accuracy of robotic seed placement between a phantom and cadaver experiment [122]. The phantom experiment demonstrated placement of seeds closer to the intended position than seen in the cadaver experiment. This was likely due to different needle-tissue interactions between the two test objects. The cadaver better represents the complex model of needle-tissue interactions. That said, the mechanical tissue properties of a cadaver are still not representative of the in-vivo situation where the tissue is lubricated. This is a feature that, to the authors knowledge, is not modelled in phantom designs for radiotherapy and is identified as an issue by a partner urologists of the phantom design in a study by Hungr et al. [116]. Whilst a simple phantom fails to model the complexity of soft tissue, the 
results may still be of benefit if they demonstrate a relative improvement of the technique.

The choice of phantom material is important for accurate dosimetry; however, the recommendations in the literature are not always followed. An example is the use of PMMA instead of the recommended plastic water for LDR in the design of an eye plaque brachytherapy phantom $[11,16]$, using ${ }^{103} \mathrm{Pd}$ source. However, the authors acknowledged the correction of PMMA to liquid water by including it in the uncertainty analysis. Similarly, the dimensions of the PMMA phantom designed by Gholami et al. were used to test the agreement of TG43 TPS calculations to Gafchromic film measurements and were smaller $(18 \times 16 \times 18 \mathrm{~cm})$ than that considered necessary for water equivalence with an ${ }^{192} \mathrm{Ir}$ source (radius $>10 \mathrm{~cm}$ ) [50]. These are considerations that the reader should be aware of when interpreting the results.

There is a need to improve the tissue mimicking materials available for brachytherapy in order to achieve substitutes, which meet the three requirements of radiative, imaging, and mechanical properties. A report that was commonly cited in the papers reviewed was ICRU report 44 [144] published in 1989, which would benefit from a later edition. Progress in manufacturing deformable phantoms can be drawn from external beam phantom development. An example is the ADAM phantom designed for CT and MRI of the whole male pelvis, focusing on mimicking the imaging and radiative properties of tissue, with organ motion from bladder and rectal filling [145]. The authors identified a peak in the CT number spectrum, which is not present in patients, likely caused by high $\mathrm{kV}$ absorption materials (PMMA or silicon); therefore, further research into alternative materials would be beneficial. Consideration is needed when using any high atomic number material at lower energies, where the photoelectric effect dominates due to the cross-section of the photoelectric being approximately proportional to $Z^{3}$. Research is also ongoing in the field of material science, and its findings will contribute to brachytherapy phantoms development. A recent example from advanced material technologies is the publication on $3 \mathrm{D}$ printing organ models with physical properties of tissue [146]. It is likely that future phantom advancement will continually use 3D printing, also referred to as "rapid prototyping". We found just a few examples of this in the review $[33,54,86,126]$, which may be a limitation of the search criteria in excluding patient specific phantoms, where 3D printing was gradually used in brachytherapy [147].

This present review considered more complex phantoms, excluding those requiring little to no manufacturing, such as slabs of solid water or simple water bath phantoms. This may cause some limitation of the study from a wider perspective of all possible phantoms; however, it focuses on more relevant designs, which have been reported in the literature.

\section{Conclusions}

In this paper, information on brachytherapy phantoms developed over the last 20 years were collected and can be used in aiding future phantom designs for departments or commercial companies. A substantial body of work has been published on phantom designs from the brachytherapy community, but a standardized method of reporting technical aspects of the phantoms is lacking. In-house phantom development demonstrates an increasing interest in MRI tissue mimicking materials, which is not yet reflected in the commercial phantoms available for brachytherapy.

Studying phantom design provides insight into the way, in which brachytherapy practice has changed over time and demonstrates customized and broad nature of the treatments offered. Phantoms provide possibility of overall quality assurance and specific quality control of the brachytherapy process; however, further development and improvement are required to keep pace with rapidly evolving clinical and scientific techniques.

\section{Acknowledgements}

The authors would like to thank the European Regional Development Fund, co-financing the CoBra project and within this, $\mathrm{PhD}$ research of which this paper is a part, under Interreg 2 Seas Mers Zeeën, from January 2018 to September 2022.

\section{Disclosure}

The authors report no conflict of interest.

\section{References}

1. https://www.ncbi.nlm.nih.gov/mesh/?term=imaging +phantom.

2. Tanderup K, Kirisits C, Damato AL. Treatment delivery verification in brachytherapy: Prospects of technology innovation. Brachytherapy 2018; 17: 1-6.

3. Doyle AJ, King DM, Browne JE. A review of the recommendations governing quality assurance of ultrasound systems used for guidance in prostate brachytherapy. Phys Med 2017; 44: 51-57.

4. Palmer AL, Bradley DA, Nisbet A. Dosimetric audit in brachytherapy. Br J Radiol 2014; 87: 20140105.

5. Pasler M, Hernandez V, Jornet $\mathrm{N}$ et al. Novel methodologies for dosimetry audits: Adapting to advanced radiotherapy techniques. Phys Imaging Radiat Oncol 2018; 5: 76-84.

6. Xu XG. An exponential growth of computational phantom research in radiation protection, imaging, and radiotherapy: A review of the fifty-year history. Phys Med Biol 2014; 59: R233-R302.

7. Ubrich F, Wulff J, Engenhart-Cabillic R et al. Correction factors for source strength determination in HDR brachytherapy using the in-phantom method. Z Med Phys 2014; 24: 138-152.

8. Wallace RE. Empirical dosimetric characterization of model iodine brachytherapy source in phantom. Med Phys 2000; 27: 2796-2802

9. Ghiassi-Nejad M, Jafarizadeh M, Ahmadian-Pour MR et al. Dosimetric characteristics of 192Ir sources used in interstitial brachytherapy. Appl Radiat Isot 2001; 55: 189-195.

10. Reniers B, Vynckier S, Scalliet P. Dosimetric study of a new palladium seed. Appl Radiat Isot 2002; 57: 805-811.

11. Sadeghi M, Hosseini SH, Raisali G. Experimental measurements and Monte Carlo calculations of dosimetric parameters of the IRA1-103Pd brachytherapy source. Appl Radiat Isot 2008; 66: 1431-1437. 
12. Arbabi A, Sadeghi M, Joharifard M. Irradiation and dosimetry of Nitinol stent for renal artery brachytherapy. Appl Radiat Isot 2009; 67: 129-132.

13. Gueli AM, Mannino G, Troja SO et al. 3D dosimetry on Ru106 plaque for ocular melanoma treatments. Radiat Meas 2011; 46: 2014-2019.

14. Mason J, Al-Qaisieh B, Bownes P et al. Monte Carlo investigation of I-125 interseed attenuation for standard and thinner seeds in prostate brachytherapy with phantom validation using a MOSFET. Med Phys 2013; 40: 031717.

15. Cohen GN, Munro JJ, Kirov A et al. 32P Brachytherapy conformal source model RIC-100 for high-dose-rate treatment of superficial disease: Monte Carlo calculations, diode measurements, and clinical implementation. Int J Radiat Oncol Biol Phys 2014; 88: 746-752.

16. Saidi P, Sadeghi M, Tenreiro C. Experimental measurements and Monte Carlo calculations for 103Pd dosimetry of the 12 mm COMS eye plaque. Phys Med 2013; 29: 286-294.

17. Chiu-Tsao ST, Medich D, Munro J. The use of new GAFCHROMIC $®$ EBT film for I125 seed dosimetry in Solid Water ${ }^{\circledR}$ phantom. Med Phys 2008; 35: 3787-3799.

18. Uniyal SC, Sharma SD, Naithani UC. A dosimetry method in the transverse plane of HDR Ir-192 brachytherapy source using gafchromic EBT2 film. Phys Med 2012; 28: 129-133.

19. Nath R, Yue N. Dose distribution along the transverse axis of a new 125I source for interstitial brachytherapy. Med Phys 2000; 27: 2536-2540.

20. Gearheart DM, Drogin A, Sowards K et al. Dosimetric characteristics of a new (125)I brachytherapy source. Med Phys 2000; 27: 2278-2285.

21. Uniyal SC, Naithani UC, Sharma SD. Evaluation of Gafchromic EBT2 film for the measurement of anisotropy function for high-dose-rate 192Ir brachytherapy source with respect to thermoluminescent dosimetry. Rep Pract Oncol Radiother 2011; 16: 14-20.

22. Jamalludin Z, Jong WL, Abdul Malik R et al. Characterization of MOSkin detector for in vivo dose verification during Cobalt-60 high dose-rate intracavitary brachytherapy. Phys Med 2019; 58: 1-7.

23. Hurley C, McLucas C, Pedrazzini G et al. High-resolution gel dosimetry of a HDR brachytherapy source using normoxic polymer gel dosimeters: Preliminary study. Nucl Instruments Methods Phys Res Sect A Accel Spectrometers Detect Assoc Equip 2006; 565: 801-811.

24. Carrara M, Gambarini G, Borroni M et al. Fricke gel dosimetric catheters in high dose rate brachytherapy. in phantom dose distribution measurements of a 5 catheter implant. Radiat Meas 2011; 46: 1924-1927.

25. Wang R, Ribouton J, Pittet $P$ et al. Implementation of GaN based real-time source position monitoring in HDR brachytherapy. Radiat Meas 2014; 71: 293-296.

26. Pittet P, Jalade P, Balosso J et al. Dosimetry systems based on Gallium Nitride probe for radiotherapy, brachytherapy and interventional radiology. IRBM 2015; 36: 92-100.

27. Broisman A, Shani G. Application of spherical micro diodes for brachytherapy dosimetry. Radiat Meas 2011; 46: 334-339.

28. Oliveira ML, Caldas LVE. Performance of thin CaSO4: Dy pellets for calibration of a $90 \mathrm{Sr}+90 \mathrm{Y}$ source. Nucl Instruments Methods Phys Res Sect A Accel Spectrometers Detect Assoc Equip 2007; 580: 293-295.

29. Brun T, Bachaud JM, Graff-Cailleaud P et al. New approach of ultra-focal brachytherapy for low- and intermediate-risk prostate cancer with custom-linked I-125 seeds: A feasibility study of optimal dose coverage. Brachytherapy 2018; 17 : 544-555.

30. Nath R, Bongiorni P, Chen $Z$ et al. Development of a rat solid tumor model for continuous low-dose-rate irradiation studies using $125 \mathrm{I}$ and $103 \mathrm{Pd}$ sources. Brachytherapy 2004; 3: 159-172.

31. Citrin D, Ning H, Guion P et al. Inverse treatment planning based on MRI for HDR prostate brachytherapy. Int J Radiat Oncol Biol Phys 2005; 61: 1267-1275.

32. Bernard S, Reniers B, Scalliet P et al. Optimization of a breast implant in Brachytherapy PDR. Validation with Monte Carlo simulation and measurements with TLDs and GafChromic films. Radiother Oncol 2005; 76: 326-333.

33. Ryu B, Bax J, Edirisinge C et al. Prostate brachytherapy with oblique needles to treat large glands and overcome pubic arch interference. Int J Radiat Oncol Biol Phys 2012; 83: 1463-1472.

34. Roué A, Ferreira IH, Van Dam J et al. The EQUAL-ESTRO audit on geometric reconstruction techniques in brachytherapy. Radiother Oncol 2006; 78: 78-83.

35. Elfrink RJ, Kolkman-Deurloo IK, Van Kleffens HJ et al. Determination of the accuracy of implant reconstruction and dose delivery in brachytherapy in The Netherlands and Belgium. Radiother Oncol 2001; 59: 297-306.

36. Roué A, Venselaar JLM, Ferreira IH et al. Development of a TLD mailed system for remote dosimetry audit for $192 \mathrm{Ir}$ HDR and PDR sources. Radiol Oncol 2007; 83: 86-93.

37. Palmer AL, Lee C, Ratcliffe AJ et al. Design and implementation of a film dosimetry audit tool for comparison of planned and delivered dose distributions in high dose rate (HDR) brachytherapy. Phys Med Biol 2013; 58: 6623-6640.

38. Diez P, Aird EGA, Sander T et al. A multicentre audit of HDR/PDR brachytherapy absolute dosimetry in association with the INTERLACE trial (NCT015662405). Phys Med Biol 2017; 62: 8832-8849.

39. Johansson B, Persson E, Westman G et al. Phantom study of radiation doses outside the target volume brachytherapy versus external radiotherapy of early breast cancer. Radiother Oncol 2003; 69: 107-112.

40. Candela-Juan C, Gimeno-Olmos J, Pujades MC et al. Fetal dose measurements and shielding efficiency assessment in a custom setup of 192 Ir brachytherapy for a pregnant woman with breast cancer. Phys Med 2015; 31: 286-292.

41. Kirisits C, Georg D, Wexberg P et al. Determination and application of the reference isodose length (RIL) for commercial endovascular brachytherapy devices. Radiother Oncol 2002; 64: 309-315.

42. Bansal AK, Semwal MK, Arora D et al. A phantom study on bladder and rectum dose measurements in brachytherapy of cervix cancer using FBX aqueous chemical dosimeter. Phys Med 2013; 29: 368-373.

43. Mantaj P, Zwierzchowski G. Measurement verification of dose distributions in pulsed-dose rate brachytherapy in breast cancer. Rep Pract Oncol Radiother 2013; 18: 139-147.

44. Nikoofar A, Hoseinpour Z, Rabi Mahdavi S et al. Highdose-rate (192)Ir brachytherapy dose verification: a phantom study. Iran J Cancer Prev 2015; 8: e2330.

45. Zwierzchowski G, Malicki J, Skowronek J. Dosimetric verification of dose optimisation algorithm during endovascular brachytherapy of the peripheral vessels. Rep Pract Oncol Radiother 2009; 14: 114-121.

46. Uniyal SC, Sharma SD, Naithani UC. Dosimetric verification of a high dose rate brachytherapy treatment planning system in homogeneous and heterogeneous media. Phys Med 2013; 29: 171-177.

47. Zhang H, Das IJ. Dosimetric perturbations at high-Z interfaces with high dose rate 192Ir source. Phys Med 2014; 30: 782-790.

48. Huh HD, Kim WC, Loh JJK et al. Rectum dose analysis employing a multi-purpose brachytherapy phantom. Jpn J Clin Oncol 2007; 37: 391-398.

49. Gerardy I, Ródenas J, van Dycke M et al. Dosimetric characterization of a brachytherapy applicator using MCNP5 
modelisation and in-phantom measurements. Appl Radiat Isot 2010; 68: 735-737.

50. Gholami S, Mirzaei HR, Arfaee AJ et al. Dose distribution verification for GYN brachytherapy using EBT Gafchromic film and TG-43 calculation. Rep Pract Oncol Radiother 2016; 2: 480-486.

51. Persson M, Nilsson J, Carlsson Tedgren Å. Experience of using MOSFET detectors for dose verification measurements in an end-to-end 192Ir brachytherapy quality assurance system. Brachytherapy 2018; 17: 227-233.

52. Flühs D, Wilke C, Naber C et al. The influence of guiding equipment and stents on the beta dose distribution in the brachytherapy of in-stent restenosis. Cardiovasc Radiat Med 2001; 2: 241-245.

53. Fazli Z, Sadeghi M, Zahmatkesh MHM et al. Dosimetric comparison between three dimensional treatment planning system, Monte Carlo simulation and gel dosimetry in nasopharynx phantom for high dose rate brachytherapy. J Cancer Res Ther 2013; 9: 402-409.

54. Silva RMV, Belinato W, Macedo LE et al. Anthropomorphic phantom to investigate the bladder dose in gynecological high-dose-rate brachytherapy. Brachytherapy 2015; 14: $633-$ 641.

55. Anton M, Wagner D, Selbach $\mathrm{HJ}$ et al. In vivo dosimetry in the urethra using alanine/ESR during (192)Ir HDR brachytherapy of prostate cancer - a phantom study. Phys Med Biol 2009; 54: 2915-2931.

56. Jeang $\mathrm{EH}, \mathrm{Goh} \mathrm{Y}, \mathrm{Cho} \mathrm{KH}$ et al. Two-dimensional in vivo rectal dosimetry during high-dose-rate brachytherapy for cervical cancer: a phantom study. Acta Oncol (Madr) 2018; 57: 1359-1366.

57. Kim J, Koo J, Choi SH et al. A preliminary study on a real-time dose monitoring system based on scintillating fibers for brachytherapy. Nucl Instruments Methods Phys Res Sect A Accel Spectrometers Detect Assoc Equip 2019; 929: 50-56.

58. Bloemen-van Gurp EJ, Murrer LHP, Haanstra BKC et al. In vivo dosimetry using a linear Mosfet-array dosimeter to determine the urethra dose in $125 \mathrm{I}$ permanent prostate implants. Int J Radiat Oncol Biol Phys 2009; 73: 314-321.

59. Gambarini G, Carrara M, Tenconi C et al. Online in vivo dosimetry in high dose rate prostate brchytherapy with MOSkin detectors: In phantom feasibility study. Appl Radiat Isot 2013; 83: 222-226.

60. Tenconi C, Carrara M, Borroni M et al. TRUS-probe integrated MOSkin detectors for rectal wall in vivo dosimetry in HDR brachytherapy: In phantom feasibility study. Radiat Meas 2014; 71: 379-383.

61. Van Gellekom MPR, Canters RAM, Dankers FJWM et al. In vivo dosimetry in gynecological applications - a feasibility study. Brachytherapy 2018; 17: 146-153.

62. Hoekstra N, Fleury E, Merino Lara TR et al. Long-term risks of secondary cancer for various whole and partial breast irradiation techniques. Radiother Oncol 2018; 128: 428-433.

63. Lee B, Ahn SH, Kim H et al. Secondary cancer-incidence risk estimates for external radiotherapy and high-dose-rate brachytherapy in cervical cancer: Phantom study. J Appl Clin Med Phys 2016; 17: 124-132.

64. Bharat S, Kung C, Dehghan E et al. Electromagnetic tracking for catheter reconstruction in ultrasound-guided highdose-rate brachytherapy of the prostate. Brachytherapy 2014; 13: 640-650.

65. Schmid M, Crook JM, Batchelar D et al. A phantom study to assess accuracy of needle identification in real-time planning of ultrasound-guided high-dose-rate prostate implants. Brachytherapy 2013; 12: 56-64.

66. Yan P, Cheeseborough JC, Chao KSC. Automatic shapebased level set segmentation for needle tracking in 3-D
TRUS-guided prostate brachytherapy. Ultrasound Med Biol 2012; 38: 1626-1636

67. Beaulieu L, Racine E, Han DY et al. Real-time electromagnetic tracking-based treatment platform for high-dose-rate prostate brachytherapy: Clinical workflows and end-to-end validation. Brachytherapy 2018; 17: 103-110.

68. Smith RL, Haworth A, Panettieri V et al. 3D catheter reconstruction in HDR prostate brachytherapy for pre-treatment verification using a flat panel detector. Phys Med 2017; 39: 121-131.

69. Wills R, Lowe G, Inchley D et al. Applicator reconstruction for HDR cervix treatment planning using images from $0.35 \mathrm{~T}$ open MR scanner. Radiother Oncol 2010; 94: 346-352.

70. Kim Y, Muruganandham M, Modrick JM et al. Evaluation of artifacts and distortions of titanium applicators on 3.0-tesla MRI: Feasibility of titanium applicators in MRI-guided brachytherapy for gynecological cancer. Int J Radiat Oncol Biol Phys 2011; 80: 947-955.

71. Aubry JF, Cheung J, Morin $\mathrm{O}$ et al. Investigation of geometric distortions on magnetic resonance and cone beam computed tomography images used for planning and verification of high-dose rate brachytherapy cervical cancer treatment. Brachytherapy 2010; 9: 266-273.

72. Schindel J, Muruganandham M, Pigge FC et al. Magnetic resonance imaging (MRI) markers for MRI-guided highdose-rate brachytherapy: Novel marker-flange for cervical cancer and marker catheters for prostate cancer. Int J Radiat Oncol Biol Phys 2013; 86: 387-393.

73. van Heerden LE, Gurney-Champion OJ, van Kesteren Z et al. Quantification of image distortions on the Utrecht interstitial CT/MR brachytherapy applicator at 3T MRI. Brachytherapy 2016; 15: 118-126.

74. Popowski Y, Hiltbrand E, Joliat D et al. Open magnetic resonance imaging using titanium-zirconium needles: Improved accuracy for interstitial brachytherapy implants? Int J Radiat Oncol Biol Phys 2000; 47: 759-765.

75. Soliman AS, Elzibak A, Easton $\mathrm{H}$ et al. Quantitative MRI assessment of a novel direction modulated brachytherapy tandem applicator for cervical cancer at $1.5 \mathrm{~T}$. Radiother Oncol 2016; 120: 500-506.

76. Roeske JC, Lund C, Pelizzari CA et al. Reduction of computed tomography metal artifacts due to the Fletcher-Suit applicator in gynecology patients receiving intracavitary brachytherapy. Brachytherapy 2003; 2: 207-214.

77. Xia D, Roeske JC, Yu L et al. A hybrid approach to reducing computed tomography metal artifacts in intracavitary brachytherapy. Brachytherapy 2005; 4: 18-23.

78. Elzibak AH, Kager PM, Soliman A et al. Quantitative CT assessment of a novel direction-modulated brachytherapy tandem applicator. Brachytherapy 2018; 17: 465-475.

79. Mehrtash A, Damato A, Pernelle G et al. EM-navigated catheter placement for gynecologic brachytherapy: an accuracy study. Proc SPIE Int Soc Opt Eng 2014; 9036: 9036-90361F.

80. Zheng D, Todor DA. A novel method for accurate needle-tip identification in trans-rectal ultrasound-based highdose-rate prostate brachytherapy. Brachytherapy 2011; 10: 466-473.

81. Mutic S, Grigsby PW, Low DA et al. PET-guided three-dimensional treatment planning of intracavitary gynecologic implants. Int J Radiat Oncol Biol Phys 2002; 52: 1104-1110.

82. Haack S, Nielsen SK, Lindegaard JC et al. Applicator reconstruction in MRI 3D image-based dose planning of brachytherapy for cervical cancer. Radiother Oncol 2009; 91: 187-193.

83. Strasmann G, Kolotas C, Heyd R et al. Navigation system for interstitial brachytherapy. Radiother Oncol 2000; 56: 49-57. 
84. Zhou J, Sebastian E, Mangona V et al. Real-time catheter tracking for high-dose-rate prostate brachytherapy using an electromagnetic 3D-guidance device: A preliminary performance study. Med Phys 2013; 40: 021716

85. Damato AL, Viswanathan AN, Don SM et al. A system to use electromagnetic tracking for the quality assurance of brachytherapy catheter digitization. Med Phys 2014; 41: 101702.

86. Lugez E, Sadjadi H, Joshi CP et al. Improved electromagnetic tracking for catheter path reconstruction with application in high-dose-rate brachytherapy. Int J Comput Assist Radiol Surg 2017; 12: 681-689.

87. Brennen T, Cutajar DL, Alnaghy S et al. BrachyView: Reconstruction of seed positions and volume of an LDR prostate brachytherapy patient plan using a baseline subtraction algorithm. Phys Med 2019; 66: 66-76.

88. Tutar IB, Managuli R, Shamdasani V et al. Tomosynthesis-based localization of radioactive seeds in prostate brachytherapy. Med Phys 2003; 30: 3135-3142.

89. McNabb E, Wong R, Noseworthy MD. Differentiating platinum coated brachytherapy seeds and gold fiducial markers with varying off-resonant frequency offsets. Magn Reson Imaging 2019; 60: 68-75.

90. Nosrati R, Soliman A, Safigholi H et al. MRI-based automated detection of implanted low dose rate (LDR) brachytherapy seeds using quantitative susceptibility mapping (QSM) and unsupervised machine learning (ML). Radiother Oncol 2018; 129: 540-547.

91. Fatemi-Ardekani A, Borg J. SU-D-213CD-05: Identifying prostate brachytherapy seeds at MRI: a study in phantom. Med Phys 2012; 39: 3618.

92. Alnaghy S, Cutajar DL, Bucci JA et al. BrachyView: Combining LDR seed positions with transrectal ultrasound imaging in a prostate gel phantom. Phys Med 2017; 34: 55-64.

93. Ding M, Wei Z, Gardi L et al. Needle and seed segmentation in intra-operative $3 \mathrm{D}$ ultrasound-guided prostate brachytherapy. Ultrasonics 2006; 44: e331-e336.

94. Jain A, Deguet A, Iordachita I et al. Intra-operative 3D guidance and edema detection in prostate brachytherapy using a non-isocentric C-arm. Med Image Anal 2012; 16: 731-743.

95. Hong JY, Rah JE, Suh TS et al. Overlapped seed localization in seed implant brachytherapy. Med Eng Phys 2009; 31: 261-267.

96. Siebert FA, De Brabandere M, Kirisits C et al. Phantom investigations on $\mathrm{CT}$ seed imaging for interstitial brachytherapy. Radiother Oncol 2007; 85: 316-323.

97. Nogueira LB, de Campos TPR. Radiological response of ceramic and polymeric devices for breast brachytherapy. Appl Radiat Isot 2012; 70: 663-669.

98. De Brabandere M, Kirisits C, Peeters R et al. Accuracy of seed reconstruction in prostate postplanning studied with a CT- and MRI-compatible phantom. Radiother Oncol 2006; 79: 190-197.

99. Mitri FG, Davis BJ, Urban MW et al. Vibro-acoustography imaging of permanent prostate brachytherapy seeds in an excised human prostate - preliminary results and technical feasibility. Ultrasonics 2009; 49: 389-394.

100. Dehghan E, Bharat S, Kung C et al. EM-enhanced US-based seed detection for prostate brachytherapy. Med Phys 2018; 45: 2357-2368.

101. Al-Qaisieh B, Smith DW, Brearley E et al. Comprehensive I-125 multi-seed comparison for prostate brachytherapy: Dosimetry and visibility analysis. Radiother Oncol 2007; 84: 140-147.

102. Blake CC, Elliot TL, Slomka PJ et al. Variability and accuracy of measurements of prostate brachytherapy seed position in vitro using three-dimensional ultrasound: An intraand inter-observer study. Med Phys 2000; 27: 2788-2795.
103. Mangili P, Stea L, Cattani F et al. Comparative study of permanent interstitial prostate brachytherapy post-implant evaluation among seven Italian institutes. Radiother Oncol 2004; 71: 13-21.

104. Kirisits C, Siebert FA, Baltas D et al. Accuracy of volume and DVH parameters determined with different brachytherapy treatment planning systems. Radiother Oncol 2007; 84: 290-297.

105. Dhanesar SK, Lim TY, Du W et al. Evaluation of the MIM Symphony treatment planning system for low-dose-rate prostate brachytherapy. J Appl Clin Med Phys 2015; 16: 62-75.

106. Kollaard RP, Dries WJF, Van Kleffens HJ et al. Recommendations on detectors and quality control procedures for brachytherapy beta sources. Radiother Oncol 2006; 78: 223-229.

107. Espinoza A, Beeksma B, Petasecca M et al. The feasibility study and characterization of a two-dimensional diode array in 'magic phantom' for high dose rate brachytherapy quality assurance. Med Phys 2013; 40: 111702.

108. Espinoza A, Petasecca M, Fuduli I et al. The evaluation of a 2D diode array in 'magic phantom'; for use in high dose rate brachytherapy pretreatment quality assurance. Med Phys 2015; 42: 663-673.

109. Kohr P, Siebert F. Quality assurance of brachytherapy afterloaders using a multi-slit phantom. Phys Med Biol 2007; 52: N387-N391.

110. Leong B, Ostyn M, Oh S et al. Technical Note: The design, construction, and evaluation of a liquid-based single phantom solution for TG128 brachytherapy ultrasound QA. Med Phys 2018; 46: 1024-1029.

111. De Almeida CE, Rodriguez M, Vianello E et al. An anthropomorphic phantom for quality assurance and training in gynaecological brachytherapy. Radiother Oncol 2002; 63: 75-81.

112. Mangold CA, Rijnders A, Georg D et al. Quality control in interstitial brachytherapy of the breast using pulsed dose rate: Treatment planning and dose delivery with an Ir-192 afterloading system. Radiother Oncol 2001; 58: 43-51.

113. Guiral P, Ribouton J, Jalade P et al. Design and testing of a phantom and instrumented gynecological applicator based on GaN dosimeter for use in high dose rate brachytherapy quality assurance. Med Phys 2016; 43: 5240-5251.

114. Pittet $P$, Jalade $P$, Gindraux L et al. DoRGaN: development of quality assurance and quality control systems for high dose rate brachytherapy based on GaN dosimetry probes. IRBM 2018; 39: 279-290.

115. Kellermeier M, Herbolzheimer J, Kreppner S et al. Electromagnetic tracking (EMT) technology for improved treatment quality assurance in interstitial brachytherapy. J Appl Clin Med Phys 2017; 18: 211-222.

116. Hungr N, Long JA, Beix V et al. A realistic deformable prostate phantom for multimodal imaging and needle-insertion procedures. Med Image Anal 2012; 39: 2031-2041.

117. Lehmann T, Rossa C, Usmani N et al. Deflection modeling for a needle actuated by lateral force and axial rotation during insertion in soft phantom tissue. Mechatronics 2017; 48: 42-53.

118. Sadjadi H, Hashtrudi-Zaad K, Fichtinger G. Needle deflection estimation: prostate brachytherapy phantom experiments. Int J Comput Assist Radiol Surg 2014; 9: 921-929.

119. Fichtinger G, Fiene J, Kennedy CW et al. Robotic assistance for ultrasound guided prostate brachytherapy. In: Lecture Notes in Computer Science (including subseries Lecture Notes in Artificial Intelligence and Lecture Notes in Bioinformatics) 2007; pp. 119-127.

120. Long JA, Hungr N, Baumann M et al. Development of a novel robot for transperineal needle based interventions: focal therapy, brachytherapy and prostate biopsies. J Urol 2012; 188: 1369-1374. 
121. Baumann M, Bolla M, Daanen V et al. Prosper: Image and robot-guided prostate brachytherapy. IRBM 2011; 32: 63-65.

122. Zhu JH, Wang J, Wang YG et al. Prospect of robotic assistance for fully automated brachytherapy seed placement into skull base: Experimental validation in phantom and cadaver. Radiother Oncol 2019; 131: 160-165.

123. Thaker NG, Kudchadker RJ, Swanson DA et al. Establishing high-quality prostate brachytherapy using a phantom simulator training program. Radiat Oncol Biol 2014; 90: 579-586.

124. Roumeliotis M, Quirk S, Skarsgard M et al. Development and characterisation of an anthropomorphic breast phantom for permanent breast seed implant brachytherapy credentialing. Brachytherapy 2018; 17: 506-513.

125. Roumeliotis M, Quirk S, Husain S et al. Establishing a simulation-based education program for radiation oncology learners in permanent seed implant brachytherapy: Building validation evidence. Brachytherapy 2020; 19: 812-819.

126. Nattagh $K$, Siauw T, Pouliot J et al. A training phantom for ultrasound-guided needle insertion and suturing. Brachytherapy 2014; 13: 413-419.

127. Loo KJ, Jakubek J, Zemlicka J et al. BrachyView: Feasibility study into the application of Timepix detectors for soft tissue thickness imaging in prostate brachytherapy treatment. Radiat Measurement 2014; 71: 329-332.

128. Gong L, Cho PS, Han BH et al. Ultrasonography and fluoroscopic fusion for prostate brachytherapy dosimetry. Int J Radiat Oncol Biol Phys 2002; 54: 1322-1330.

129. Mizowaki T, Cohen GN, Fung AYC et al. Towards integrating functional imaging in the treatment of prostate cancer with radiation: The registration of the MR spectroscopy imaging to ultrasound/CT images and its implementation in treatment planning. Int I Radiat Oncol Biol Phys 2002; 54: 1558-1564.

130. Carlsson $\AA$, Carlsson GA. Influence of phantom material and dimensions on experimental 192 Ir dosimetry. Med Phys 2009; 36: 2228-2235.

131. Schoenfeld AA, Harder D, Poppe B et al. Water equivalent phantom materials for192Ir brachytherapy. Phys Med Biol 2015; 60: 9403-9420.

132. Schoenfeld AA, Thieben M, Harder D. Evaluation of water-mimicking solid phantom materials for use in HDR and LDR brachytherapy dosimetry. Phys Med Biol 2017; 62: N561-N572.

133. Sina S, Lotfalizadeh F, Karimipourfard M et al. Material-specific conversion factors for different solid phantoms used in the dosimetry of different brachytherapy sources. Iran J Med Phys 2015; 12

134. Pfeiffer D, Sutlief S, Feng W et al. AAPM Task Group 128: Quality assurance tests for prostate brachytherapy ultrasound systems. Med Phys 2008; 35: 5471-5489.

135. Meigooni AS, Gearheart DM, Sowards K. Experimental determination of dosimetric characteristics of Best ${ }^{\circledR} 125 \mathrm{I}$ brachytherapy source. Med Phys 2000; 27: 2168-2173.

136. Nath R, Anderson LL, Luxton G et al. Dosimetry of interstitial brachytherapy sources: Recommendations of the AAPM Radiation Therapy Committee Task Group No. 43. Med Phys 1995; 2: 209-234.

137. Rivard MJ, Coursey BM, DeWerd LA et al. Update of AAPM Task Group No. 43 Report: A revised AAPM protocol for brachytherapy dose calculations. Med Phys 2004; 31: 633-674.

138. Rivard MJ, Butler WM, DeWerd LA et al. Supplement to the 2004 update of the AAPM Task Group No. 43 Report. Med Phys 2007; 34: 2187-2205.

139. Pittet P, Lu GN, Galvan JM et al. Implantable real-time dosimetric probe using GaN as scintillation material. Sens Actuators A Phys 2009; 151: 29-34.
140. CIRS. Tissue Equivalent Ultrasound Prostate Phantom. https://www.cirsinc.com/products/ultrasound/zerdinehydrogel/tissue-equivalent-ultrasound-prostate-phantom/.

141. Muench P, Meigooni AS, Nath R et al. Photon energy dependence of the sensitivity of radiochromic film and comparison with silver halide film and LiF TLDs used for brachytherapy dosimetry. Med Phys 1991; 18: 769-775.

142. Farhood B, Geraily G, Abtahi SMM. A systematic review of clinical applications of polymer gel dosimeters in radiotherapy. Appl Radiat Isot 2019; 143: 47-59.

143. Siebert FA, Kirisits C, Hellebust TP et al. GEC-ESTRO/ ACROP recommendations for quality assurance of ultrasound imaging in brachytherapy. Radiother Oncol 2020; 148: 51-56.

144. International Commission on Radiation Units and Measurements. Tissue substitutes in radiation dosimetry and measurement. 1989

145. Niebuhr NI, Johnen W, Echner G et al. The ADAM-pelvis phantom-an anthropomorphic, deformable and multimodal phantom for MRgRT. Phys Med Biol 2019; 64: 04NT05.

146. Qiu K, Zhao Z, Haghiashtiani G et al. 3D printed organ models with physical properties of tissue and integrated sensors. Adv Mater Technol 2018; 3: 1700235.

147. Choi CH, Kim J, Park JM. A 3D-printed patient-specific applicator guide for use in high-dose-rate interstitial brachytherapy for tongue cancer: a phantom study. Phys Med Biol 2019; 64: 135002. 